\title{
Field-based DGTD/PIC technique for general and stable simulation of interaction between light and electron bunches
}

\author{
Arya Fallahi ${ }^{1}$ and Franz Kärtner ${ }^{1,2,3,4}$ \\ ${ }^{1}$ Center for Free-Electron Laser Science, DESY, Notkestrasse 85, D-22607 Hamburg, Germany \\ ${ }^{2}$ Department of Physics, University of Hamburg, Jungiusstrasse 9, D-20355 Hamburg, Germany \\ ${ }^{3}$ The Hamburg Center for Ultrafast Imaging, Luruper Chaussee 149, D-22761 Hamburg, Germany \\ ${ }^{4}$ Department of Electrical Engineering and Computer Science and Research Laboratory of Electronics, \\ Massachusetts Institute of Technology, 77 Massachusetts Avenue, Cambridge, Massachusetts 02139, USA \\ E-mail: arya.fallahi@cfel.de
}

Received 29 May 2014, revised 7 August 2014

Accepted for publication 19 August 2014

Published DD MM 2014

\begin{abstract}
We introduce a hybrid technique based on the discontinuous Galerkin time domain (DGTD) and the particle in cell (PIC) simulation methods for the analysis of interaction between light and charged particles. The DGTD algorithm is a three-dimensional, dual-field and fully explicit method for efficiently solving Maxwell equations in the time domain on unstructured grids. On the other hand, the PIC algorithm is a versatile technique for the simulation of charged particles in an electromagnetic field. This paper introduces a novel strategy for combining both methods to solve for the electron motion and field distribution when an optical beam interacts with an electron bunch in a very general geometry. The developed software offers a complete and stable numerical solution of the problem for arbitrary charge and field distributions in the time domain on unstructured grids. For this purpose, an advanced search algorithm is developed for fast calculation of field data at charge points and for later importing to the PIC simulations. In addition, we propose a field-based coupling between the two methods resulting in a stable and precise time marching scheme for both fields and charged particle motion. To benchmark the solver, some examples are numerically solved and compared with analytical solutions. Eventually, the developed software is utilized to simulate the field emission from a flat metal plate and a silicon nano-tip. In the future, we will use this technique for the simulation and design of ultrafast compact $\mathrm{X}$-ray sources.
\end{abstract}

Keywords: electron acceleration, numerical simulation, particle in cell, discontinuous Galerkin method, field emission

(Some figures may appear in colour only in the online journal)

\section{Introduction}

The interaction of charged particles with an electromagnetic field occurs in many applications and devices, ranging from radiation sources [1, 2] to accelerator physics [3, 4], imaging science and spectroscopy [5]. Many of today's high power sources such as microwave traveling wave tubes [6], X-band magnetrons [7], synchrotron radiation sources, THz and X-ray free electron lasers $[1,2]$ are performing based on free electron motion in a properly designed electromagnetic field. In accelerator physics, energy transfer to particles is achieved by the action of an electromagnetic wave, either in a cavity or a waveguide. The mutual effects between electrons and a laser beam constitute the fundamentals for developing advanced sources based on inverse compton scattering [8, 9] and undulator radiation $[1,2,10]$. In the latter case, not only the action of the field on an electron bunch is studied but also the back-action of free charges on the field distribution. This type of interaction also plays a major role in the promising laser plasma wake-field acceleration [11, 12]. 
Due to the broad range of applications various algorithms are developed to solve charged particle interaction with an electromagnetic wave. A widely used technique considers the stream of charges as a current density in the Maxwell's equations and solves for the fields and the electric current simultaneously [13]. This task can be incorporated in many standard algorithms like finite element method (FEM), finite difference time domain (FDTD) as well as method of moments, and is available in some of the existing commercial software packages [14]. However, the method treats charge distribution macroscopically and is not capable of studying the internal charge profile evolutions during the interaction. Hydrodynamic models for the electron bunch based on distribution functions are developed to mitigate this problem. Direct solutions of transient distribution functions in tandem with the electromagnetic fields result in the so-called Maxwell-Vlasov equations, widely studied in the past decades $[15,16]$. A detailed description of solving Maxwell-Vlasov equations for plasmas using discontinuous Galerkin (DG) approach is presented in [17]. These developed models, although very helpful, again consider the cumulative effects of charge distributions and are able to make approximate predictions on the microscopic properties of the bunch. In contrast, with particle in cell (PIC) codes $[18,19]$ variations in the microscopic bunch parameters can be simulated and are therefore standard computational techniques for simulating beam dynamics.

In PIC simulations, the equations of motion are updated continuously for a particular electromagnetic field profile. The field distribution is obtained by either using analytical formulations or importing some previously solved numerical values. The former procedure is often followed for solving optical beams or waveguide modes interaction with charge distribution, where approximate analytical solutions (e.g. Gaussian and Bessel-Gaussian beams) are available [20-22]. Nevertheless, the accuracy of using analytical solutions for such purposes is still under debate [23]. The use of numerical field solutions is indeed a standard technique in designing accelerator cavities, where the harmonic solutions of the fields in cavities are used as input to the PIC algorithm. However, the method suffers from harsh limitations when short pulses are influencing a bunch, which has recently received substantial attention due to the possibilities opened in ultrafast optics [24] and THz sources [25]. Therefore, the time domain numerical simulation of field propagation when acting on a bunch and considering its microscopic effects is very often encountered in the domains mentioned above.

Figure 1 shows a schematic illustration for the general problem of light-electron interaction. An arbitrary optical beam excites fields inside a computational domain with existing boundary conditions, a set of scatterers and an initial charge distribution. The algorithm has to solve for the evolution of the electromagnetic fields and charge distribution. The goal is to find a solution by providing a time marching mechanism that propagates the electromagnetic fields in a computational domain and simultaneously solves for bunch evolution. Firstly, we need to decide on a rigorous time domain method for solving Maxwell's equations. FDTD is a

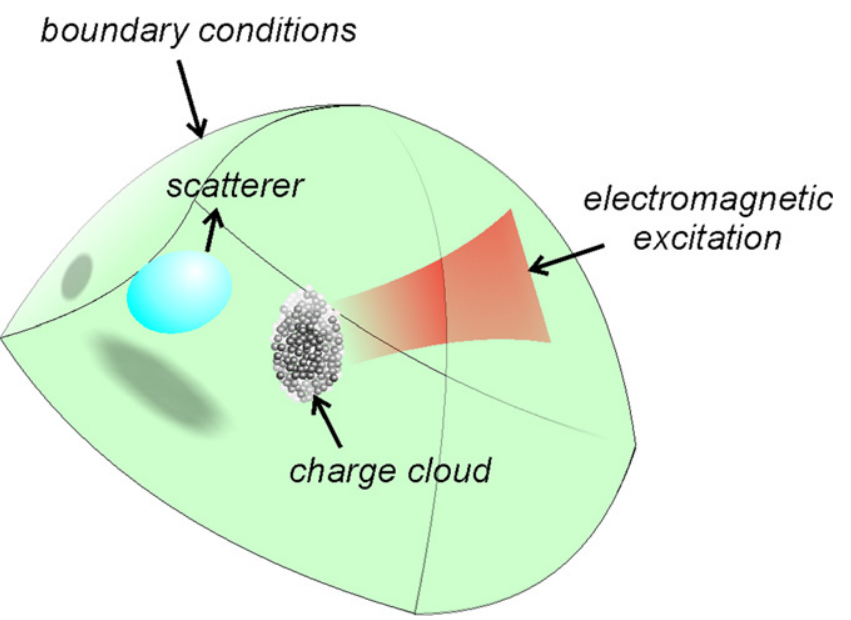

Figure 1. Schematic illustration of the general problem of light and electron interaction.

superior choice with high efficiency, versatility and flexibility [26]. Extensive research efforts have been devoted to develop FDTD/PIC codes leading to software packages like SELFT [27], MAGIC [28], MAFIA [29] and PIConGPU [30]. However, this method suffers from severe limitations being second order accurate in time and space and only amenable to uniform Cartesian grids. Techniques such as sub-gridding and split-material voxels have been proposed, without completely solving this problem. Recent years witnessed progress in the DG methods for solving time domain electromagnetic equations with high order accuracy and additionally on unstructured grids [31-35]. Therefore, we chose discontinuous Galerkin time domain (DGTD) as the Maxwell solver and due to the previously outlined reasons the particle motion is solved by a PIC algorithm leading to the hybrid DGTD/PIC algorithm.

Using DGTD as a kernel for solving Maxwell's equations and coupling it with a PIC algorithm is pioneered by Jacobs and Hesthaven [36]. The method introduces equivalent charge $\rho(\boldsymbol{r})$ and current $\boldsymbol{J}(\boldsymbol{r})$ densities, and projects them into the computational grid. It is further used to model RF accelerators and guns [37]. Nonetheless, there are several restrictions to the algorithm which makes it not suitable for problems involving ultrafast field variations and tiny charge distributions [38]. Some examples are (i) consideration of smooth functions for charge distribution to avoid Gibbs type phenomena in the field computations, (ii) numerical instabilities due to static charge and force build-up, (iii) singularities in the field solution of the cell containing the charges, and (iv) inability to distinguish between the radiated fields to avoid a charge being affected by its own radiation. These effects have been investigated extensively in various studies [36, 39] and several techniques such as hyperbolic divergence cleaning [36] are proposed to mitigate some of the aforementioned problems. In [40], advantage was taken from an exception of the above effects, which vanish on uniform Cartesian grids, to simulate plasma wake-field acceleration. This solution indeed ignores the outstanding advantage of DG approaches that is the capability of handling unstructured 
meshes, and is inefficient for problems where many different length scales are involved. The goal in the presented study is to demonstrate a numerical scheme which can model the ultrafast interactions between electrons and laser pulses and apply it to problems with non-smooth charge distributions, without observing numerical instablities, singularities in the field solution close to the particles while avoiding self-action of the particles. A more mathematical investigation of algorithmic performance of the developed code with respect to these challenges will be pursued in the future.

The methodology for implementing this algorithm is described in the next section. We start with a brief description of DGTD and PIC methods and proceed with the idea of uniform grid mapping for efficiently combining the two routines. The benefit of the proposed algorithm lies in the novel method for coupling the particle radiation into the propagating field, which is the last topic in this section. After describing each numerical method benchmarks are presented to assess the accuracy of the implementation. Two promising examples are analyzed in section 3 and finally section 4 concludes the paper.

\section{Methodology}

\subsection{DGTD method}

For the numerical calculation of field profiles in time, we employ the high order DG formulation of Hesthaven and Warburton [31, 32]. The method focuses on solving Maxwell's equations for dispersive media

$$
\nabla \times \boldsymbol{H}=\boldsymbol{J}+\boldsymbol{J}_{p}+\epsilon_{0} \frac{\partial \boldsymbol{E}}{\partial t}, \quad \text { and } \quad \nabla \times \boldsymbol{E}=-\mu_{0} \frac{\partial \boldsymbol{H}}{\partial t},
$$

where $\boldsymbol{J}_{p}=\partial \boldsymbol{P} / \partial t$ stands for the polarization current in the material and $\boldsymbol{J}=\boldsymbol{J}_{0}+\sigma \boldsymbol{E}$ represents the total flowing current. In order to solve this problem, the computational domain is tessellated into $M$ tetrahedral elements $\Omega_{m}$. In each element, the fields and currents are written as an expansion in terms of a set of presumed basis functions

$$
\boldsymbol{Q}^{m}(\boldsymbol{r}, t)=\sum_{j=1}^{N} q_{j}^{m}(t) \boldsymbol{w}_{j}(\boldsymbol{r}),
$$

where $Q \in\left\{\boldsymbol{E}, \boldsymbol{H}, \boldsymbol{J}, \boldsymbol{J}_{p}\right\}$ represents any of the involved electromagnetic quantities and $N$ is the number of coefficients determined by the order of utilized basis functions. Throughout the formulation the superscripts $m$ are used to refer to the $m$ th element. The basis functions assumed in this work are the hierarchical polynomial vector basis functions developed by Webb [41], which lead to the dependence $N=(n+1)(n+2)(n+3) / 6$, with $n$ being the order of basis functions. Therefore, one may refer to our implementation as a modal DG approach in comparison with the nodal formulation in [31]. Due to the accurate inversion of the mass matrices in the DGTD algorithm, the computational cost of nodal and modal basis functions are the same. However, the Webb basis functions have benefits in computing the coupling of fields in adjacent elements and the possibility to distinguish between the rotational and irrotational functions. The latter leads to the basis functions, well-known as expansion sets with $n+1 / 2$ orders.

The standard Galerkin method follows the weighted residual approach which satisfies the original equation based on weighted integrals. The expanded Maxwell equation (1) are multiplied with the basis functions and integrated over each element $\Omega^{m}$, which yields

$$
\begin{aligned}
\epsilon_{0} \sum_{j=1}^{N} S_{i j} \frac{\mathrm{d} e_{j}^{m}}{\mathrm{~d} t}= & \sum_{j=1}^{N} T_{i j} h_{j}^{m}+\sum_{j=1}^{N} \iint_{\partial \Omega^{m}} \boldsymbol{w}_{i} \cdot(\hat{\boldsymbol{n}} \times \boldsymbol{H}) \mathrm{d} s \\
& -\sum_{j=1}^{N} S_{i j}\left(j_{j}^{m}+j_{p j}^{m}\right) \\
\mu_{0} \sum_{j=1}^{N} S_{i j} \frac{\mathrm{d} h_{j}^{m}}{\mathrm{~d} t}= & -\sum_{j=1}^{N} T_{i j} e_{j}^{m} \\
& -\sum_{j=1}^{N} \iint_{\partial \Omega^{m}} \boldsymbol{w}_{i} \cdot(\hat{\boldsymbol{n}} \times \boldsymbol{E}) \mathrm{d} s
\end{aligned}
$$

where

$$
S_{i j}=\iiint_{\Omega^{m}} \boldsymbol{w}_{i} \cdot \boldsymbol{w}_{j} \mathrm{~d} v \text { and } T_{i j}=\iiint_{\Omega^{m}}\left(\nabla \times \boldsymbol{w}_{i} \cdot \boldsymbol{w}_{j}\right) \mathrm{d} v .
$$

As observed in the equations a surface integral appears in the weighted residual formulation which is the term responsible for the coupling with the adjacent elements. The discontinuous nature of the expansion results in different values of tangential field quantities at the surface, if calculated from the expansion in either of the elements. This discontinuity leads to the so-called numerical flux through the element surface. The clever idea of DG approaches is defining a proper surface field obtained from the values at both elements to acquire a stable and convergent scheme in which the numerical flux tends to zero. Based on the upwinding flux theory introduced in [42], the proper expression for the tangential surface fields are

$$
\begin{aligned}
\hat{\boldsymbol{n}} \times \boldsymbol{H} & =\hat{\boldsymbol{n}} \times \frac{Z^{m} \boldsymbol{H}^{m}+Z^{l} \boldsymbol{H}^{l}+\hat{\boldsymbol{n}} \times\left(\boldsymbol{E}^{m}-\boldsymbol{E}^{l}\right)}{Z^{m}+Z^{l}}, \\
\hat{\boldsymbol{n}} \times \boldsymbol{E} & =\hat{\boldsymbol{n}} \times \frac{Y^{m} \boldsymbol{E}^{m}+Y^{l} \boldsymbol{E}^{l}-\hat{\boldsymbol{n}} \times\left(\boldsymbol{H}^{m}-\boldsymbol{H}^{l}\right)}{Y^{m}+Y^{l}},
\end{aligned}
$$

where the superscript $l$ implies that the field value is extracted from the neighboring element. Further, we have introduced cell impedances $Z^{m}=\sqrt{\mu^{m} / \epsilon^{m}}$ and admittances $Y^{m}=\sqrt{\epsilon^{m} / \mu^{m}}$. Using the above definition, the inter-element coupling terms are obtained based on the fields at the local and adjacent elements. For the surfaces corresponding to particular boundary conditions such as open, perfect electric or perfect magnetic conductors, proper definitions are available and introduced in [31-34].

The developed formulation leads to explicit expressions for the time derivative of the coefficient vectors in terms of their values, i.e. the so-called initial value problem $\dot{\boldsymbol{q}}=f(\boldsymbol{q}, t)$, with $\boldsymbol{q}$ defined as a vector containing all the 

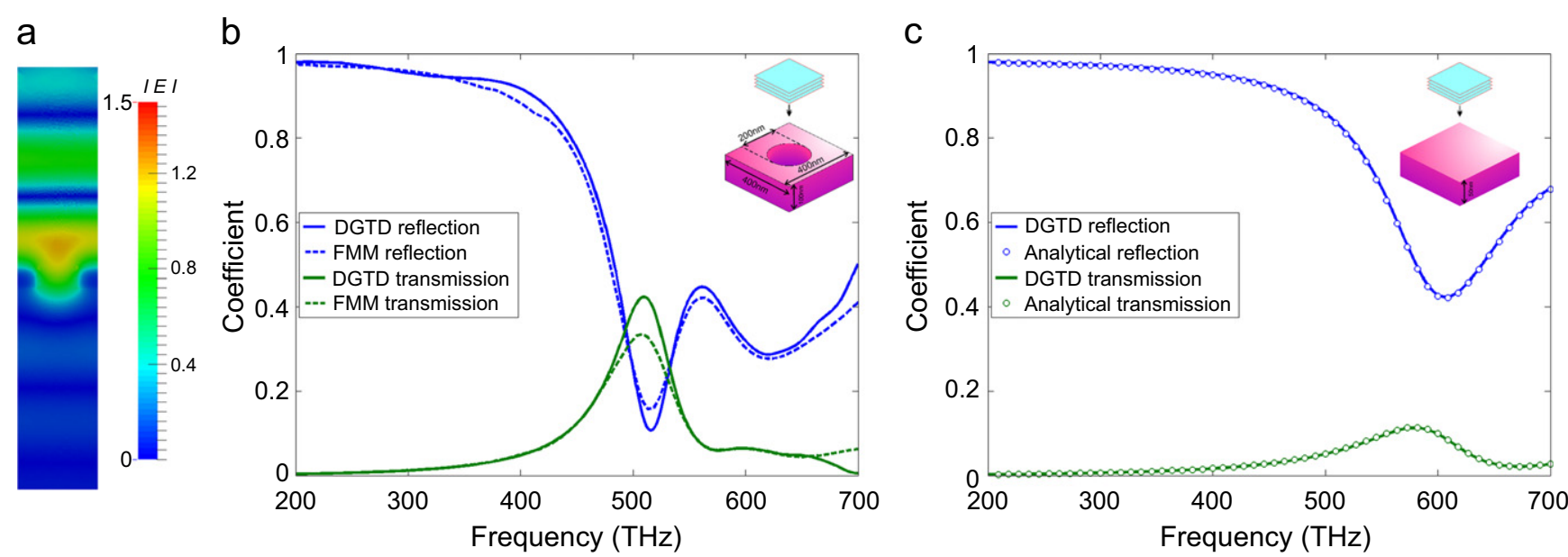

Figure 2. Normal incidence of a plane wave with Gaussian temporal variations on a gold photonic crystal slab considered for testing the DGTD code. (a) Snapshot of the obtained field profile using the DGTD code. (b) The reflection and transmission coefficient of the photonic crystal slab calculated using DGTD and FMM methods are compared. (c) The same analysis is done for a homogeneous gold slab and compared against analytical results.

coefficients for various quantities. The remaining step of integrating the semi-discrete system in time is fulfilled through a 4th order Runge-Kutta scheme. To achieve a stable time marching process the time step for the update must be less than a limit. This limit is empirically set according to the criterion introduced in [33]

$$
\delta t \leqslant \zeta \delta x / c,
$$

where $\delta x$ is the minimum cell size, $c$ the speed of light and $\zeta$ a factor set according to the basis function order which is $1,1 / 2$, $1 / 4$ and $1 / 5$ for orders from 1 to 4 , respectively.

Using the presented DGTD algorithm a general Maxwell solver is developed for solving the temporal variation of fields for arbitrary excitation and geometries. For the sake of brevity, we avoid explaining the implementation details of our DGTD software. The space integrals are computed using Gaussian quadratures properly set for the specific order of basis functions. Moreover, for considering the dispersive material properties the formulation of auxiliary differential equations is employed in conjunction with different material models, including Debye, Drude, and Lorentz. The developed DGTD software prepares a platform for propagating electromagnetic fields caused by an external excitation and the particle radiation.

As mentioned in the introduction, we examine the implementation through some benchmarks to assess its reliability and accuracy. To this end, we consider simple problems whose analytic solutions are available. For the DGTD implementation, normal incidence of a plane wave on a photonic crystal slab is analyzed. The subset of figure 2(b) shows the considered geometry of the unit cell as well as the plane-wave excitation. The temporal signature of the plane wave amplitude is assumed to be a Gaussian signal with pulse duration $3 \mathrm{fs}$ and central wavelength $400 \mathrm{~nm}$. The host material of the photonic crystal slab is assumed to be gold, whose permittivity is obtained from the Drude-Lorentz model proposed in [43]. We assume periodic boundaries on the sides of the unit cell and the two upper and lower boundaries truncate the domain through 1st order absorbing boundary conditions (ABC). The total domain is tessellated into 50083 elements, which leads to 10 as time steps and $10 \mathrm{~ms}$ computation time for each time update on an 8 core machine with Linux operating system. A screenshot of the resulting field profile is shown in figure 2(a). Furthermore, in figure 2(b), we compare the results obtained using the DGTD code and another semi-analytical method, namely Fourier modal method (FMM) developed mainly for the analysis of planar periodic structures. In the frequency range of the excitation, good agreement is observed between the results. The discrepancy at higher frequencies occurs because of low resolution of the mesh compared to the wavelength. In addition, the small difference in reflection and transmission at resonance occurs because of the slow convergence of the FMM results at resonance frequencies. To validate this, we repeat the same study for a homogenous gold slab, where analytical solutions are available for both transmission and reflection coefficients. Figure 2(c) illustrates the problem and presents numerical and analytical results, which evidence a perfect agreement between the two solutions.

\subsection{PIC method}

The PIC method is a general technique used to solve a certain class of partial differential equations encountered in various fields such as fluid dynamics and plasma physics [18]. The success of the PIC method for plasma simulations is mainly due to being relatively intuitive and straightforward to implement. The method begins with integrating the equations of motion, which for relativistic electrons reads as

$$
\begin{gathered}
\frac{\partial}{\partial t}\left(\gamma m_{0} \boldsymbol{v}(\boldsymbol{r}, t)\right)=-e\left(\boldsymbol{E}(\boldsymbol{r}, t)+\mu_{0} \boldsymbol{v} \times \boldsymbol{H}(\boldsymbol{r}, t)\right), \\
\frac{\partial \boldsymbol{r}}{\partial t}=\boldsymbol{v}(\boldsymbol{r}, t) .
\end{gathered}
$$


a

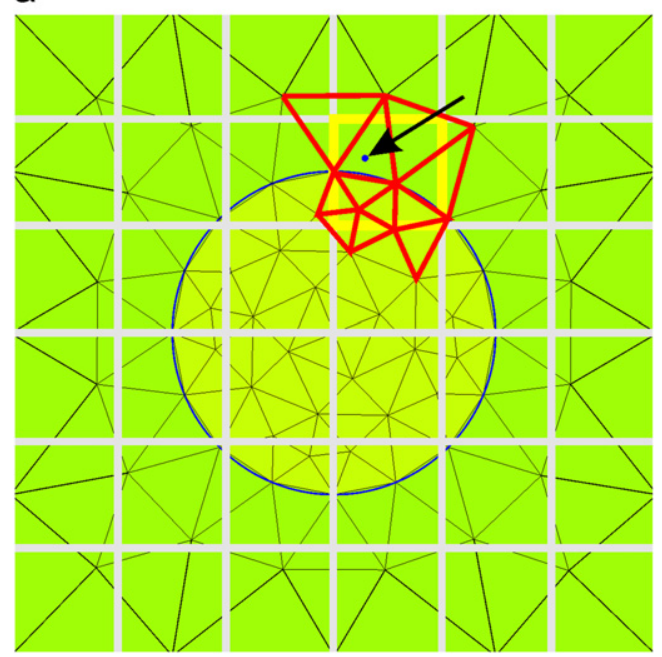

b

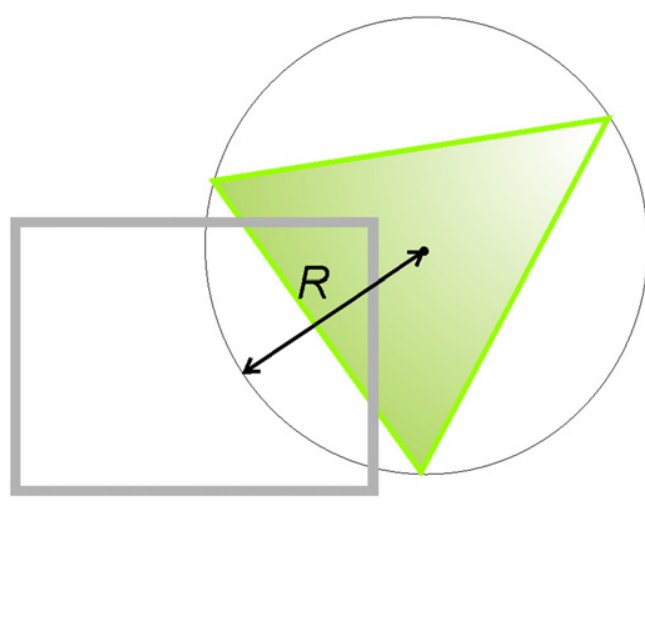

Figure 3. (a) Illustration of the uniform grid mapping algorithm in 2D: the red triangles are checked to find the triangle containing the charge location. (b) Illustration of the criterion to decide if a pixel in the uniform grid shares a region with a tetrahedral in the unstructured grid.

In traditional PIC implementations, the fields of charges are interpolated on a pre-defined mesh and then a second interpolation returns the field values at the particle locations. This leads to average field approximations for solving the particle motion $[44,45]$. The computational cost for this algorithm, known as PM (particle-mesh) method, is proportional to $N_{e}$, with $N_{e}$ being the total number of particles. The inaccuracy of the PM methods inspires the calculation of particles interaction using direct binary formulations, which are generally referred to as PP (particle-particle) models. This implementation leads to computational cost proportional to $N_{e}^{2}$. There are also models that consider both types of interactions to achieve both accuracy and calculation speed and are called PP-PM or $\mathrm{P}^{3} \mathrm{M}$ models with computation costs proportional to $N_{e} \log N_{e}$ [46].

For integrating the equations of motion, we use the same 4th order Runge-Kutta scheme as in the DGTD implementation. This synergy has the advantage of directly using the calculated field values without the need for time interpolation and memorizing the results of the previous time steps. Using a Boris scheme for time integration is also possible and has the advantage of preserving the conserved quantities. Nonetheless, different time integration schemes for PIC and DGTD necessitate field interpolation over time steps which may add to the computational cost. Reading the DGTD field profile and calculating the values at different charge locations faces an additional overhead. The main advantage of DGTD is its capability for handling various geometries, because it is developed for unstructured grids. The apparent and usual way to find the tessellation element containing the point of interest in an unstructured grid is to start checking each element based on this criterion and stop the search as soon as the corresponding element is found. Imagine the simulation takes 10000 particles (or macroparticles) into account. Additionally, the DGTD simulation contains 100000 elements. Consequently, the described algorithm requires one billion element checks in each time step, which drastically increases the computational cost. The solution to this problem is the uniform grid mapping algorithm explained as follows.

The reason for the aforementioned problem in obtaining field values lies in the unstructured nature of the spatial grid. If the space discretization would be based on a uniform hexahedral grid, the containing element could be found by using an analytical formulation leading to a much shorter computation time. The idea how to deal with an unstructured grid is by mapping it on a uniform hexahedral grid. Figure 3(a) presents a 2D illustration of this mapping technique. First, the whole 2D unstructured mesh is overlapped on a uniform rectangular grid and in a preprocessing step all the triangles sharing common regions with a specific pixel are found. This analysis provides a map that assigns each pixel in the uniform grid a set of triangles in the unstructured grid. By using a simple analytic equation, the pixel containing the location (yellow pixel in figure 3(a)) is obtained. This map returns the corresponding triangles (red triangles in figure 3(a)), which are checked to find the triangle containing the point. By following the same procedure in three dimensions, the element of interest is found after checking only few tetrahedrons, which strongly depends on the resolution of the uniform hexahedral compared to the tetrahedral grid.

An important point when constituting the map is the criterion deciding whether a tetrahedron should be maintained in the set for one hexahedron, or in the 2D case, whether a triangle should be maintained in the set of one rectangle. A conclusion based on the triangle vertices does not lead to a correct map, because as observed in figure 3(b) the two elements can share common regions without the triangle vertices residing in the rectangle. A proper conclusion is made using the radius of the circumscribed sphere (or circle in 2D). If and only if this sphere has no common region with the hexahedron, the element should be excluded from the corresponding set in the map. Using the introduced search algorithm, a fast procedure for calculating the accelerating field of a charge is 
a

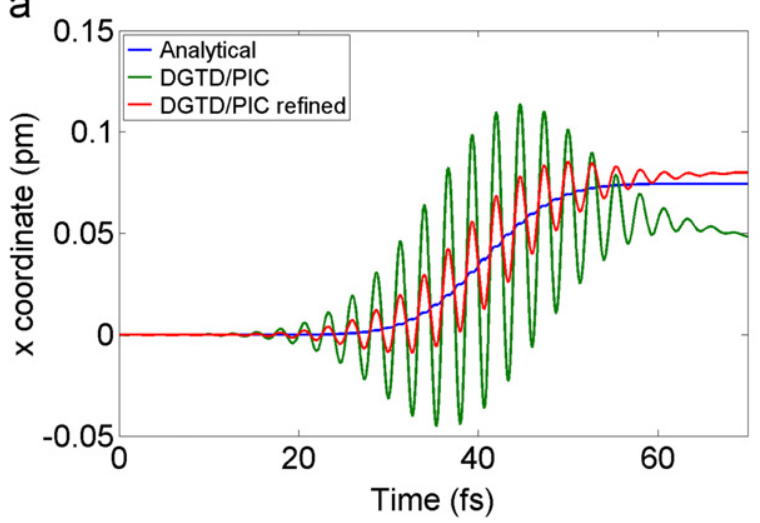

b

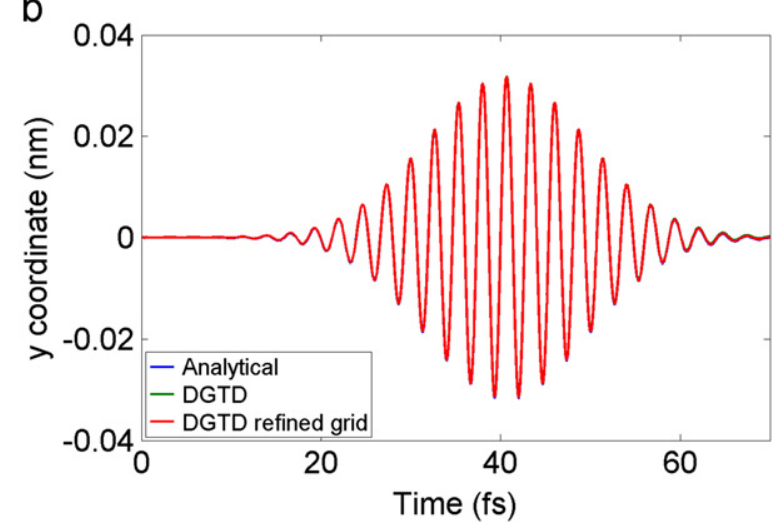

Figure 4. (a) $x(t)$ and (b) $y(t)$ of an electron residing on the coordinate origin and influenced by an incoming $y$-polarized electromagnetic plane wave. The results, obtained using the DGTD/PIC code are compared with the analytical formulation.

achieved, which leads to an efficient solution of the system in the time domain.

To benchmark the PIC code, we focus on the dynamics of a single charged particle affected by a plane wave. Since the plane wave propagation is simulated by the DGTD code, this problem implicitly serves as a benchmark for the DGTD part as well. A sphere is considered as the computational domain with 1st order $\mathrm{ABC}$ boundaries through which a $y$ polarized plane wave with Gaussian envelope enters and propagates along the $+x$-axis. The center wavelength of the incoming pulse is $800 \mathrm{~nm}$, the pulse duration is assumed to be $15 \mathrm{fs}$ and the peak field is set to $1 \mathrm{GV} \mathrm{m}^{-1}$. An electron resides on the center of the sphere and moves due to the electromagnetic fields of the plane wave.

We solve for the position of the electron using the developed DGTD/PIC solver. On the other hand, this problem can be solved analytically using the relativistic Hamiltonian of a free particle. The vector potential of the considered plane wave reads as

$$
\begin{aligned}
\boldsymbol{A}= & \int_{-\infty}^{t} E_{0} \mathrm{e}^{-2 \ln 2\left(\frac{t-\left(x-x_{0}\right) / c}{\tau}\right)^{2}} \\
& \times \cos \left(\omega\left(t-\frac{x-x_{0}}{c}\right)+\psi_{0}\right) \mathrm{d} t \hat{\boldsymbol{y}},
\end{aligned}
$$

where $E_{0}$ denotes the peak field, $\tau$ is the pulse duration and $\psi_{0}$ stands for the carrier envelope phase of the signal. The timedependent position of the electron is then obtained as the following:

$$
\begin{aligned}
& x(t)=\frac{e^{2}}{2 \gamma^{2} c m_{0}^{2}} \int_{-\infty}^{t} A_{y}^{2}(t) \mathrm{d} t, \\
& y(t)=\frac{e}{\gamma m_{0}} \int_{-\infty}^{t} A_{y}(t) \mathrm{d} t, \text { and } z(t)=0
\end{aligned}
$$

where $\gamma$ represents the Lorentz factor corresponding to the instantaneous energy of the electron. The motion along the $y$ axis happens due to the electric field of the plane wave, and the ponderomotive force triggers the motion along the $x$-axis. In figure 4, we plot and compare the temporal evolution of functions $x(t)$ and $y(t)$ obtained using both the analytical formulation and the DGTD/PIC algorithm. A perfect agreement is observed for the variations in $y$ coordinate. The motion along $x$ is a second order effect due to the ponderomotive force. As seen from figure 4, the total amount of particle movement is thousand times less than the wavelength of the plane wave. Accurate prediction of this small motion requires very high resolution in the space discretization resulting in large computation cost. In figure 4, results obtained with one step refinement of the DGTD mesh are also illustrated. The comparison of the obtained tajectorys evidences the convergence of the results toward the analytical solution. As deduced from the curves the DGTD/PIC code is able to predict the small ponderomotive motion of the electron along the $x$-axis with $10 \%$ error, which demonstrates the high accuracy of the algorithm and its reliability.

\subsection{Particle radiation}

In addition to accounting for the field effects on particles' motion, a powerful PIC code should also consider the reverse effect, namely the effect of particle motion on the fields. This is always a challenge in the simulation using PIC methods. The various versions of Lagrangian methods (PM, PP and $\mathrm{P}^{3} \mathrm{M}$ ) tackle this problem in different ways to achieve either high accuracy or low computational cost. A common issue in all these models is neglecting the time delay needed by the charge fields to travel through the mesh points. The reasons are the very large memory requirements and consequently hampering of the computations for considering this effect. This was the motivation for developing semi-Lagrangian techniques like FDTD/PIC and DGTD/PIC methods.

The DGTD/PIC algorithm offers here an advantage, which is the possibility of considering mutual interactions with the time delay effect without making spatial interpolation of the fields. Moreover, it gives an attractive framework to take into account boundary effects on the radiation from the moving charge. All these goals are achieved only by additional computational cost proportional to the number of particles $N_{e}$. The traditional approach to include the particle interactions is to expand the effective charge and current distribution

$$
\left(\rho(\boldsymbol{r}, t)=\sum_{j=1}^{N} q \delta\left(\boldsymbol{r}-\boldsymbol{r}_{i}\right)\right.
$$
and 

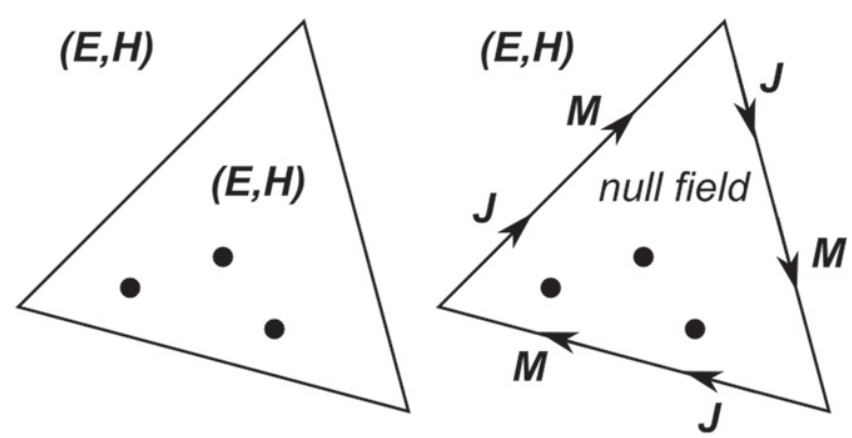

Figure 5. Illustration of the equivalence principle used to couple the radiated field into the propagating fields of the DGTD code.

$\left.\boldsymbol{J}(\boldsymbol{r}, t)=\sum_{j=1}^{N} q \boldsymbol{v}_{i} \delta\left(\boldsymbol{r}-\boldsymbol{r}_{i}\right)\right)$ in terms of basis functions in equation (2). The problems in this method emanate from the fact that the DGTD algorithm tackles solving Maxwell's equation (1), where the charge conservation is not considered and the discontinuous nature of the field expansions create parasitic charges in the field solutions. These effects are further amplified when real charges are moving in the domain. The problems encountered with this technique have been briefly described in the introductory section. The method, which we are presenting here, couples the charge motion inherently with the Maxwell solver through the radiated fields from the charge. This results in a drastic softening of the parasitic effects.

To couple the radiated field of a moving particle to the propagating fields of the DGTD, we exploit the equivalence principle in electromagnetics. Suppose there are a number of charged particles residing in an element, as illustrated in figure 5. For the region outside the element, this set of charge points can be replaced with a surrounding boundary $S_{f}$ on which a particular magnetic and dielectric current is flowing. These currents are to be found from the radiated field according to

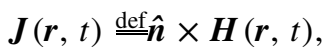

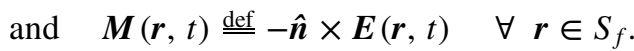

where $\boldsymbol{H}$ and $\boldsymbol{E}$ are the instantaneous radiated field of the charges inside the element. We consider $S_{f}$ to be the boundary of one element. The fields inside the element according to the above currents will then be zero. A basic assumption of FEMs is the homogeneity of the medium in each discretization element. Therefore, the radiated fields are not affected by any boundary before reaching the element surfaces. Note that in this step, we have neglected the required travel time for the particle radiation to reach the element boundaries. This is justified based on the very small size of the elements compared to the radiation wavelength. Consequently, $\boldsymbol{H}$ and $\boldsymbol{E}$ can be derived from the Liénard-Wichert potential. Note, that no free space assumption is considered here. After coupling to propagating fields, it will be reflected or transmitted by the boundaries around the charges. The above assumptions lead to correct results for the radiation as long as the elements containing the charges and accordingly the time step size are small enough. It is well-known that high order DGTD can use elements with large sizes compared to wavelength. In such cases, the time delay for the radiated field within a single cell becomes important. In the next section, the procedure to account for this effect is briefly described.

The outstanding benefit of this technique is that one can distinguish between the static fields and the propagating fields. In other words, only the propagating fields (sometimes referred to as wakefield) of the moving particle are considered in (10) and not the static field. This leads to a divergenceless solution of the field with small excitation of the parasitic effects as observed in the conventional algorithm. Therefore, $\boldsymbol{E}$ and $\boldsymbol{H}$ in (10) can be written as

$$
\begin{aligned}
\boldsymbol{E}(\boldsymbol{r}, t)= & \sum_{i} \boldsymbol{E}_{i}(\boldsymbol{r}, t) \\
= & \sum_{i} \frac{q_{i}}{4 \pi \epsilon_{0}\left(1-\left(\boldsymbol{\beta}_{i} . \hat{\boldsymbol{d}}_{i}\right)\right)^{3}} \\
& \times\left(\frac{\hat{\boldsymbol{d}}_{i}\left(\boldsymbol{\beta}_{i} . \hat{\boldsymbol{d}}_{i}\right)-\boldsymbol{\beta}_{i}}{\gamma_{i}^{2}\left|\boldsymbol{r}-\boldsymbol{r}_{i}\right|^{2}}+\frac{\hat{\boldsymbol{d}}_{i} \times\left(\left(\hat{\boldsymbol{d}}_{i}-\boldsymbol{\beta}_{i}\right) \times \dot{\boldsymbol{\beta}}_{i}\right)}{c\left|\boldsymbol{r}-\boldsymbol{r}_{i}\right|}\right), \\
\boldsymbol{H}(\boldsymbol{r}, t)= & \sum_{i} \frac{\hat{\boldsymbol{d}}_{i} \times \boldsymbol{E}_{i}(\boldsymbol{r}, t)}{Z_{m}},
\end{aligned}
$$

where $\boldsymbol{r}_{i}, \boldsymbol{\beta}_{i}$ and $\gamma_{i}$ denote the position vector, normalized velocity and Lorentz factor of the charge $q_{i}$, respectively. $\hat{\boldsymbol{d}}_{i}=\left(\boldsymbol{r}-\boldsymbol{r}_{i}\right) /\left|\boldsymbol{r}-\boldsymbol{r}_{i}\right|$ and $Z_{m}$ is the intrinsic impedance in the element. In order to inject the defined surface currents in (10) to the DGTD calculations, one needs to calculate the tangential fields on the elements using the following modified relation [47]

$$
\begin{aligned}
& \hat{\boldsymbol{n}} \times \boldsymbol{H}=\hat{\boldsymbol{n}} \\
& \quad \times \frac{Z^{m} \boldsymbol{H}^{m}+Z^{l} \boldsymbol{H}^{l}-Z^{l} \boldsymbol{J}+\hat{\boldsymbol{n}} \times\left(\boldsymbol{E}^{m}-\boldsymbol{E}^{l}\right)-\boldsymbol{M}}{Z^{m}+Z^{l}}, \\
& \hat{\boldsymbol{n}} \times \boldsymbol{E}=\hat{\boldsymbol{n}} \\
& \quad \times \frac{Y^{m} \boldsymbol{E}^{m}+Y^{l} \boldsymbol{E}^{l}+Y^{l} \boldsymbol{M}-\hat{\boldsymbol{n}} \times\left(\boldsymbol{H}^{m}-\boldsymbol{H}^{l}\right)-\boldsymbol{J}}{Y^{m}+Y^{l}} .
\end{aligned}
$$

Thus a discontinuous field is added to the DGTD results, which is equal to the radiation field outside the element and zero inside. By following the presented procedure, the radiated field of charges outside the containing element is calculated and propagated. However, if an element contains more than one charge point, the above formulation ignores the interaction between the particles inside one element. For calculating the interaction between particles within an element, we use the point-to-point formulation. In this case, the total field (propagating plus static field) is considered.

The presented formulation gives a complete description for implementing the field-based DGTD/PIC algorithm in order to solve for laser-electron interactions. Before discussing the pros and cons of this method, we investigate the reliability of the method through a simple benchmark. We consider a single particle with $-1.6 \mathrm{fC}$ charge initially at rest 


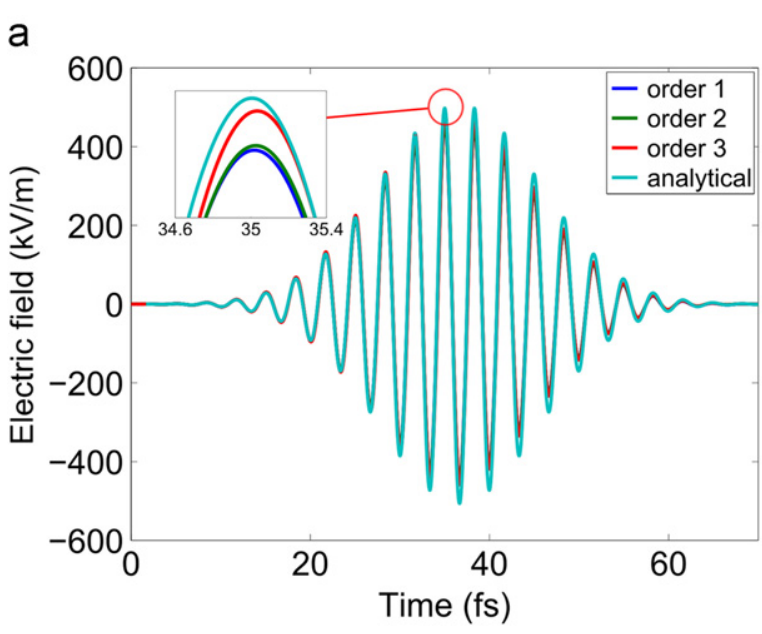

b

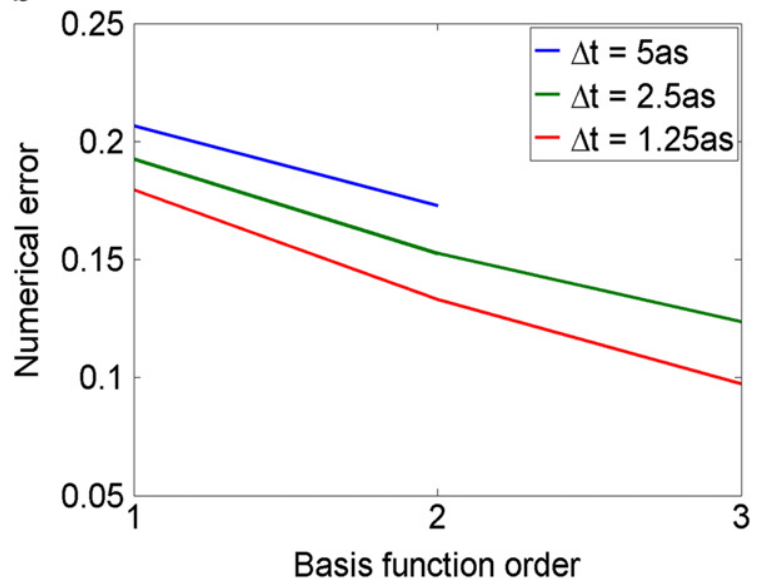

C

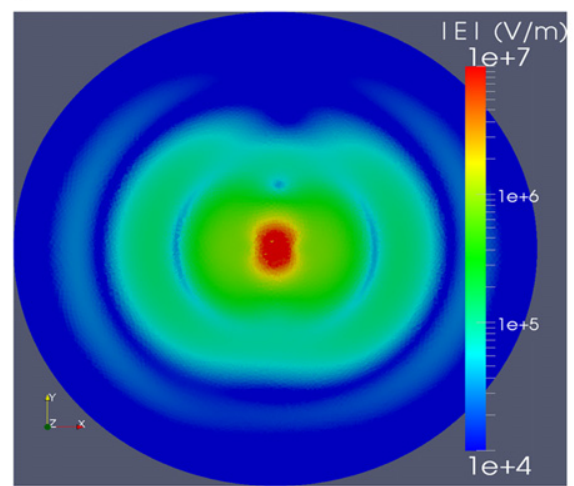

Figure 6. Field-based DGTD/PIC results for the radiation field of one particle accelerated by a laser beam: (a) the field sampled at the point $(0.45,0.0,0.0) \mu \mathrm{m}$ is compared with analytic calculations, (b) the numerical error with respect to the analytical solution is plotted for different orders and time steps, and (c) the magnitude of the electric field profile of the radiation in the propagation plane of the incident plane-wave.

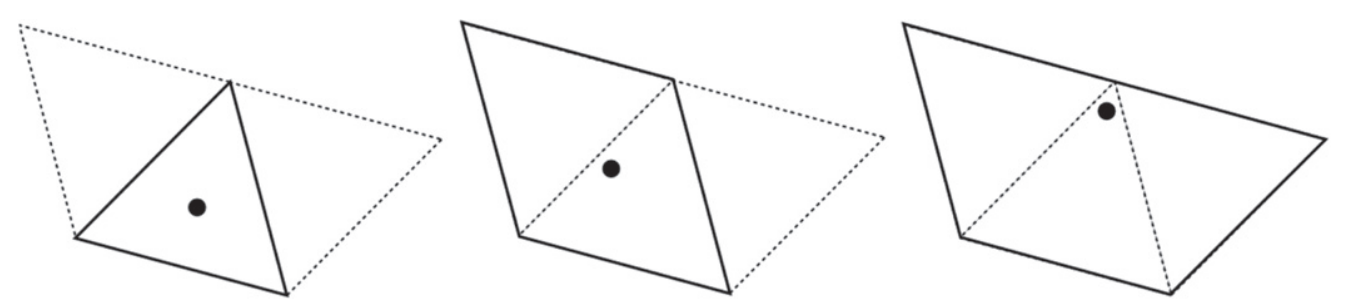

Figure 7. Illustration of surface selection for projecting the particle radiation into the propagating fields: the solid lines represent the surface on which the equivalent currents are calculated and added to the fields.

affected by a plane-wave with Gaussian temporal profile (pulse duration $=15 \mathrm{fs}$, peak electric field $=10 \mathrm{GV} \mathrm{m}^{-1}$, and central wavelength $=1 \mu \mathrm{m}$ ), and solve for the particle radiation. If the small spatial vibration of the particle is neglected, this problem can be solved analytically and serves as a proper benchmark for the assessment of the particle radiation computation. Nevertheless, this problem is very challenging for any numerical scheme based on spatial discretizations. Due to the assumption of the point charge, there is a large inhomogenity of the radiated field on the element boundaries. Therefore, assuming high order basis functions (which is a unique feature of the DGTD technique) is helpful in this case. Normally, the problems involve interaction of light with charge distributions, which produce smooth fields on the element faces and remove the above difficulty. Figure 6(a) shows the sampled electric field at $450 \mathrm{~nm}$ distance from the particle computed using the field-based DGTD/PIC method with different orders and the analytic calculation. Good agreement is found, as well as zero static force build-up in the numerical scheme. We define the numerical error based on $e=\sum\left|E_{n}-E_{a}\right| / \sum\left|E_{a}\right|$, with $E_{a}$ the analytical solution and $E_{n}$ the numerically solved field. The calculated error in terms of basis function order and the considered time step is plotted in figure 6(b). In figure 6(c), the field profile of the radiation is illustrated, which is free from usually observed parasitic effects. 


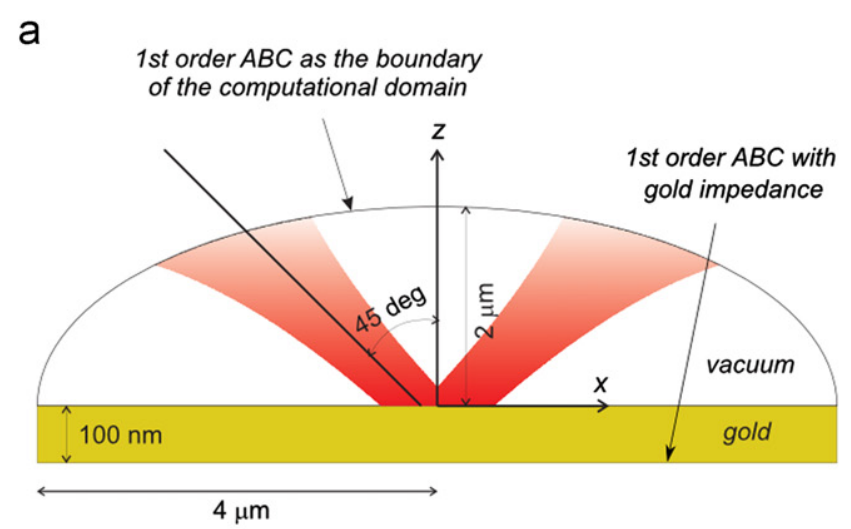

b

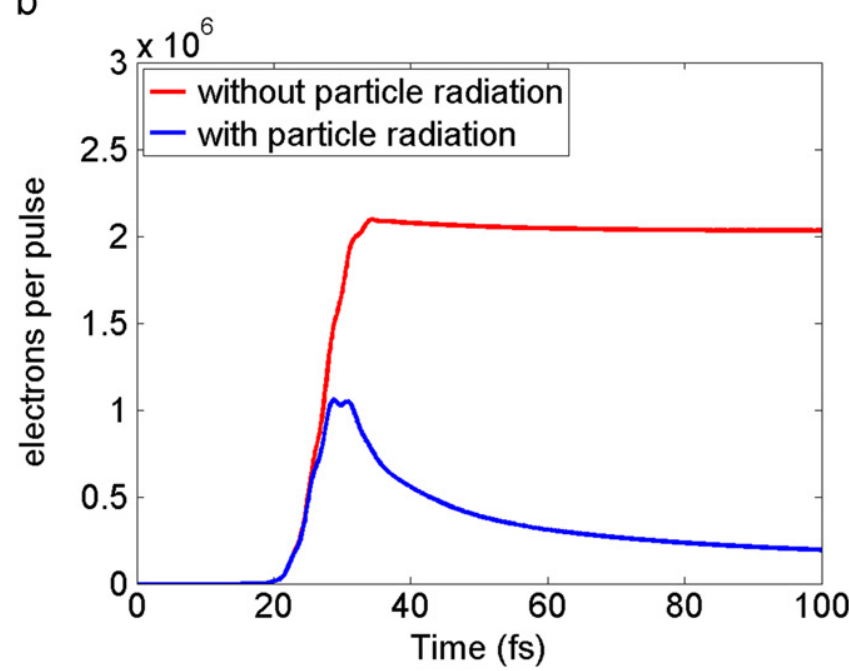

C

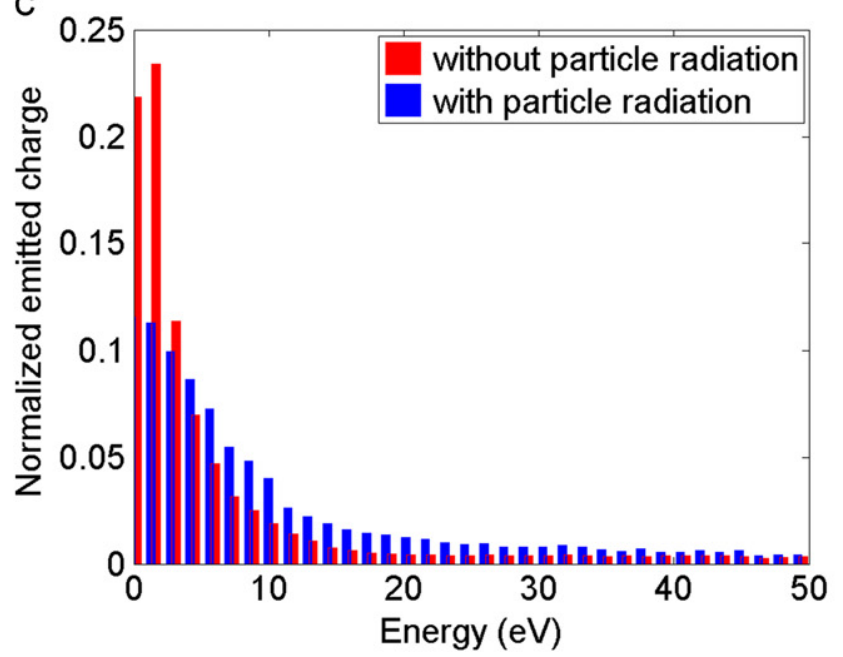

Figure 8. (a) Geometry of the field-emitter cathode to be studied: a short pulse laser beam impinges on a flat gold plate and simultaneously accelerates the emitted electrons. (b) Charge emitted from the flat surface versus time compared for calculations with and without considering particle radiation. (c) The final energy profile of the emitted charge for both calculations.

\subsection{Discussion of the field-based DGTD/PIC method}

Like any other computational method, the developed fieldbased DGTD/PIC has advantages and drawbacks, which are discussed in the following. First, there is an additional computational cost due to the assumption of particle radiation. The total cost added to the computation is obtained as:

total computaion cost $=$ cost of calculating the fields on the element surface + cost of calculating the point - to - point interactions in one element

$$
=N_{e}+M+\sum_{m=1}^{M} N_{c m}^{2} \cong N_{e}+M,
$$

where $M$ is the total number of elements containing at least one charge. $N_{c m}$ stands for the number of charges in the $m$ th element and is proportional to the total number of charges divided by the total number of elements, i.e. $N_{c} / M$. Since the numbers $N_{c}$ and $M$ are usually similar, the cost introduced by the PP calculations is negligible compared to the radiation calculations.

From the analytic formulation, it is apparent that the particles never get affected by their own radiation. However, due to the numerical descritization of the particle radiation, the field claculated due to the surrounding currents inside the element (figure 5) may not be exactly zero. This effect is minimized through high order expansions, and thereby more accurate numerical integration of the fields on the element surfaces. The same hypothesis applies to the static fields, meaning that the divergence of the radiated field will not be exactly zero due to numerical errors. However, it will be only a small field because the source of static fields are removed from the solution.

Following the formulation of field-based DGTD/PIC algorithms, one needs to calculate integrals of the form,

$$
F\left(\boldsymbol{r}_{s}\right)=\int_{\boldsymbol{r} \in \partial \Omega} \frac{f\left(\boldsymbol{r}, \boldsymbol{r}-\boldsymbol{r}_{s}\right)}{\left|\boldsymbol{r}-\boldsymbol{r}_{s}\right|^{n}} \mathrm{~d} s,
$$

which are known as weakly singular integrals, because the radiation source (particle) can be very close to the surface, which in turn introduces nearly singular terms to the integral. It is well-known that using the conventional Gaussian quadrature techniques to numerically evaluate the above integral suffers from slow convergence rates. In [48], several strategies are introduced for efficient calculation of these integrals. We have employed the introduced technique in [48] welladapted to triangular surfaces for efficient and accurate calculation of the weakly singular integrals.

Neglecting the time delay for field propagation within one element may introduce small errors to the final result. When large elements and high orders are considered, the particle radiation needs several time steps to reach the element boundary. However, taking into account the time delay effect is in that case a straightforward task. One needs to consider an array of excitation vectors for each element. The radiation contribution is then added to the $k$ th excitation vector, if the distance $d$ to the integration point satisfies $k c \delta t<d<(k+1) c \delta t$. With each particle update this array is shifted, and for field updates the first excitation vector (corresponding to the update time) is used. This method leads to good accuracy in the results without increasing computational cost at moderate increase in memory requirements. 

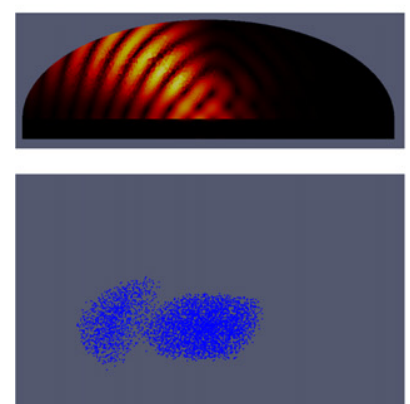

$\mathrm{T}=18 \mathrm{fs}$
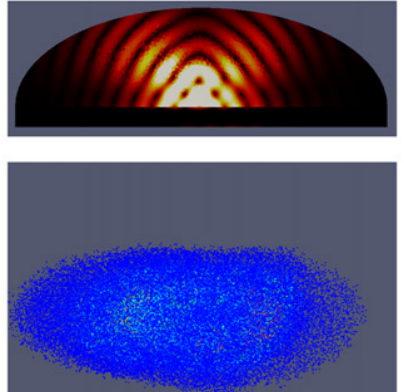

$\mathrm{T}=27 \mathrm{fs}$
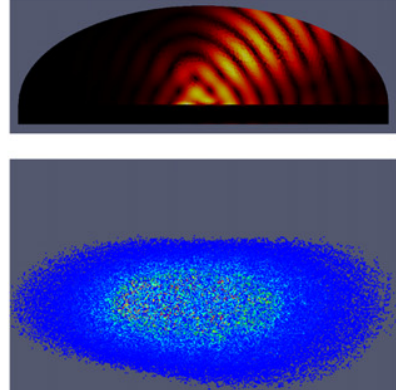

$\mathrm{T}=36 \mathrm{fs}$
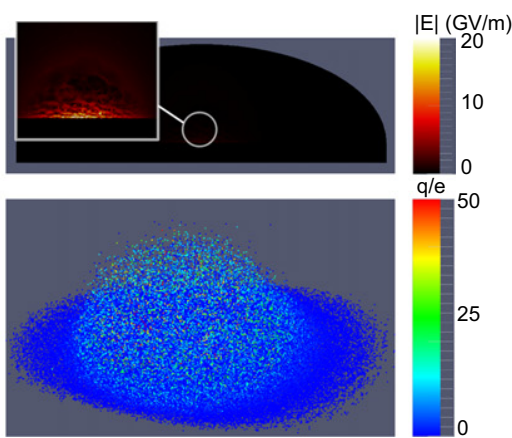

$\mathrm{T}=100 \mathrm{fs}$

Figure 9. Snapshots of the incident field and the emitted current from the flat gold emitter: the expansion of the charge cloud with time after the emission process is partially driven by space-charge effects.

a

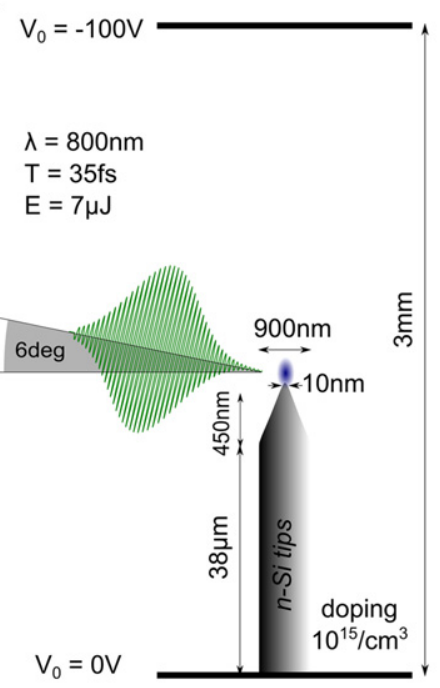

C

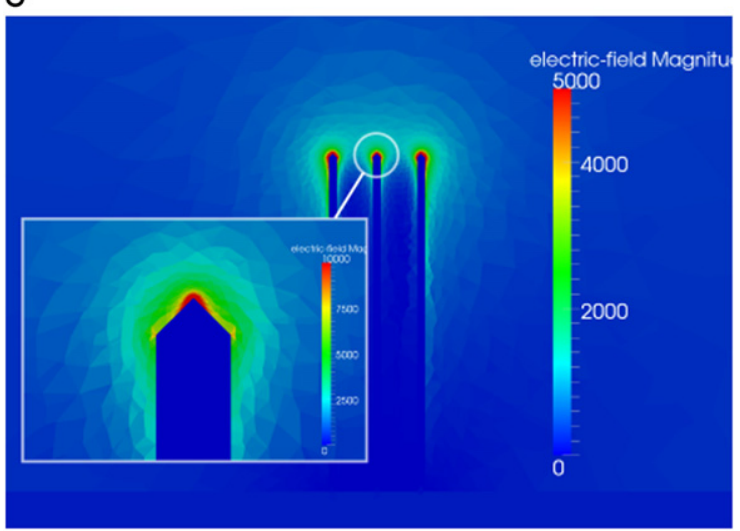

$\mathrm{b}$

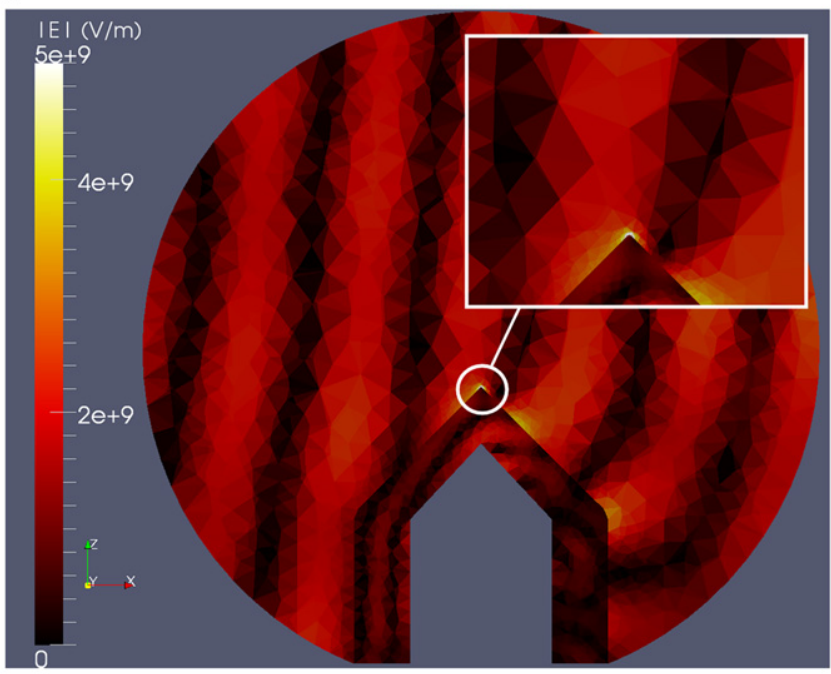

d

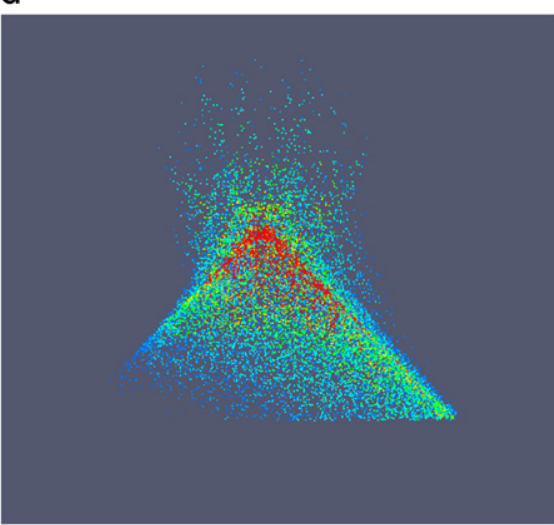

Figure 10. (a) Geometry for field emission from a silicon nanotip: a laser beam with 35 fs pulses obliquely illuminates a silicon nanotip, emits electrons and simultaneously accelerates the emitted electrons. (b) The computed field profile using the DGTD algorithm for one single tip. (c) The computed static field profile using a FEM Poisson solver for three tips. (d) Snap-shot of the emitted charge cloud from the tip.

In the current-based DGTD/PIC algorithm smooth charge and current distributions need to be used to avoid appearance of parasitic effects. In [36], an approximation of these distributions by B-spline functions is suggested. In the field-based solution, the particles affect the propagating fields through their radiation, which are inherently smooth functions, thereby avoiding this approximation. Nonetheless, both methods suffer from a phenomenon known as grid heating. Due to the motion of particles in the computaional grid, there exists abrupt variations of the field excitation, when particles enter the element. This causes excitations of the element internal resonances and the emergence of 
a

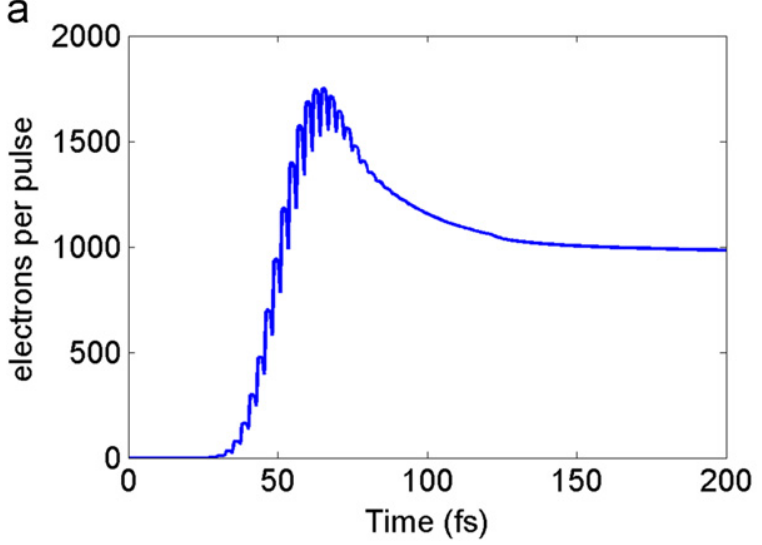

b

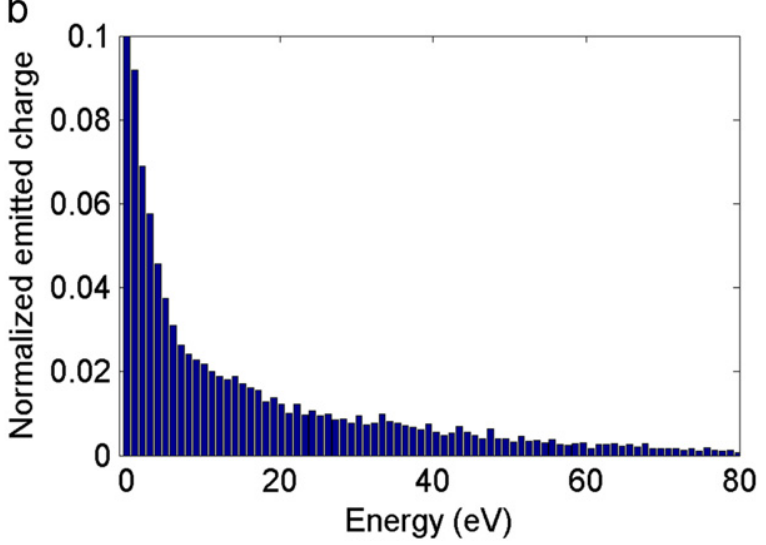

Figure 11. (a) Total emitted charge from the nanotip versus time and (b) energy distribution of the emitted particles at $t=120 f s$.

numerical artifacts. Employing a dynamic selection process for closed surfaces, on which the equivalent currents are calculated (figure 5), considerably weakens grid heating. This technique is illustrated in figure 7. The main rule is:

'If the distance from the charge point to the element face is less than a predefined value $(D)$, then a combination of both elements sharing the face should be considered as the surrounding region.'

Using this dynamic selection of surfaces assures a gradual increase in the excitation fields of one element. Avoidance of abrupt changes in the field values close to the charge in turn adds significantly to the numerical accuracy. Note that the obejctive of this paper is an introduction of the field-based DGTD/PIC method. For the sake of brevity, the detailed studies on the algorithm effciency and problems (such as numerical dispersion and numerical Cerenkov radiation) based on rigorous numerical studies will be presented in another study.

As mentioned earlier, the static fields caused by charges are not transferred over elements. Although these local static fields can be often ignored depending on the application, they play a significant role in space-charge limited processes. The associated electric fields are rapidly decaying fields and the contribution from nearest particles are usually the dominant ones. Hence, considering the static fields between charges within one element and calculating the static Ewald sum [44], provides a good estimate for space-charge effects. For better accuracy, it is suggested to account for space-charge effects due to charges in neighboring elements. Owing to the high order nature of the DGTD/PIC method, considering large elements is possible. Therefore, computation of the static fields caused by charges in the surrounding elements provides an appropriate estimate for space-charge effects.

\section{Application examples}

\subsection{Field emission from a metal plate}

The field-based DGTD/PIC algorithm is a promising method to solve complex, strong-field ultrafast electro-optical problems where no analytic solution exists. One particular problem, that is currently of high interest is the study of ultrafast electron guns utilizing laser-induced field emission. These devices, known as field-emitting cathodes, exploit quantum tunneling in the presence of strong electric fields for the generation of high brightness electron beams. Practically, an ultra-short laser pulse illuminates a bulk metal surface and extracts the electrons from the surface. The free electrons are then further affected by the existing fields and follow the corresponding trajectory in free-space. Since the field emission problem contains propagation and scattering of electromagnetic fields as well as particle motion, the DGTD/PIC algorithm is a well-suited method for simulating this phenomenon. In addition, the capability of handling unstructured grids enables one to adaptively increase the mesh resolution at the electron emission points. First, we consider the problem of field emission from a flat metal surface.

Figure 8(a) shows the considered material configuration, computational domain and the excitation film. We assume a tightly focused Gaussian laser beam with central wavelength $\lambda=800 \mathrm{~nm}$, pulse duration $\tau=10 \mathrm{fs}$, Rayleigh radius $w_{0}=500 \mathrm{~nm}$, and peak-field amplitude $E_{0}=10 \mathrm{GV} \mathrm{m}^{-1}$ that is obliquely $\left(\theta=45^{\circ}\right)$ incident on the thin gold surface. The gold layer is assumed to be a circular disc with radius $4 \mu \mathrm{m}$ and thickness $400 \mathrm{~nm}$. The whole computational domain is discretized into 82137 tetrahedra resulting in $0.6 \mathrm{~s}$ calculation time for field updates at each time step of 2 as duration. Electrons are emitted according to the Fowler-Nordheim emission model for metallic surfaces [49]. The work function is assumed to be $w=5.1 \mathrm{eV}$ and the probability for reflection of electrons on the surface, when returning back to the surface is set to $r=0.3$.

The strong laser pulse is able to extract many electrons from the surface. However, due to the mutual interaction between the particles and also the image charge effect, a considerable portion of the emitted charge recombines with the surface. This is the well-known space-charge limit in electron guns which can be simulated only correctly, if the mutual interactions are taken into account. The image charge effect emerging from the charge distribution interacting with 
the emitting surface, dictates solving Maxwell's equations including material boundaries.

Numerical modeling of this problem results in the total charge emission versus time shown in figure 8(b). As seen from the graphs, the maximum emission of charge is happening around the peak of the laser field, which affirms the standard assumptions in the field emission mechanism. We compare the field emission results with and without considering particle interactions, i.e. particle fields. According to the computation without considering particle radiated fields, the short laser pulse leads to emission of about two million electrons from the surface. However, when particle radiated fields and the mutual static interactions are considered, the Coulomb blockade effect supresses tunneling of about half of this charge. Afterwards, the attraction from image charge and mutual repulsion of electrons lead to recombination of charge with the gold surface. Note that the initial kinetic energy of the electrons immediately after tunneling is assumed to be zero similar to strong field emission from atoms [50]. The energy distributions of the emitted electrons for the two simulations are illustrated in figure 8(c). It is observed that the particle interactions result in significant broadening of energy distribution of particles and suppression of low energy electrons by recombination with the surface.

Figure 9 presents snapshots of field and charge distributions above the gold surface. The color coded dots in the charge distribution represents a charge cloud that forms in the region. Therefore, the charge of each point can be less than the charge of one electron, since it refers to the probability distribution rather than one single electron. The figure sequence shows that one side of the bunch is created earlier than the other side due to the oblique incidence of the Gaussian beam. Moreover, the zoom on the origin in the last snapshot shows the remaining space-charge fields above the surface.

\subsection{Field emission from silicon nano-tips}

In the last example, the field-based DGTD/PIC technique is utilized for the analysis of laser induced field emission from silicon nano-tips. The geometry of the problem is shown in figure 10(a). A single silicon high aspect ratio nano-tip resides on a cathode plane. A 35 fs laser pulse with center wavelength at $800 \mathrm{~nm}$ and peak field amplitude $E_{0}=4.72 \mathrm{GV} \mathrm{m}^{-1}$ (pulse energy $7 \mu \mathrm{J}$ ), illuminates the tip and causes field emission from the apex. The applied voltage between the two anode and cathode planes ( $100 \mathrm{~V}$ in this example) helps to extract the electrons over barrier created by space-charge and imagecharge, thereby enhancing the emission efficiency of the device. The silicon nanotip enhances both laser and static fields at the apex, which necessitates accurate analysis of both optical and dc field profiles. Due to the minute length scales at the tip when compared with the laser wavelength, the capability of the DGTD/PIC technique in handling unstructured grids is very helpful to achieve efficient computation. We use the FEM to solve for the static field distribution around the nano-tip. Figures 10(b)-(d) shows the obtained distributions for the static field, laser field and the emitted charge.
According to the FEM results, the static field is enhanced about 60 times at the tip. The DGTD results showed that the optical field enhancement is only about 9.5. The total emitted charge versus time and the final energy distribution of the emitted electrons are presented in figure 11. According to the results, about 1000 electrons are emitted from a single silicon nanotip after each pulse. This is in good agreement with experimental results reported in [51].

\section{Conclusion}

A new hybrid method for numerical simulation of the interaction between an electromagnetic field and a bunch of charged particles is introduced. The proposed procedure combines the DGTD and PIC methods for the analysis of combined field propagation and particle motion. A uniform grid mapping algorithm is developed to efficiently import the computed fields from the DGTD to the PIC code. Furthermore, a new strategy for the coupling of radiation from moving charges to the DGTD is introduced. The combined technique enables simulation of space-charge effects with an additional cost proportional to the total number of particles. The developed software based on the DGTD/PIC technique is verified through various benchmarks and finally applied to problems of electron field-emission from a metal cathode and nanotips. This method offers an efficient approach towards laser particle interaction and can serve as a powerful simulation tool for researchers in various fields such as particle acceleration, free-electron lasers, plasma science, spectroscopy, and electron diffractive imaging.

\section{Acknowledgements}

This work is supported by the European Research Council (ERC) under grant 609920. We would like to thank DESY HPC team for providing access and computation time at the HPC-center at DESY.

\section{References}

[1] Saldin E L, Schneidmiller E A and Yurkov M V 2000 The Physics Of Free Electron Lasers (Berlin: Springer)

[2] Schmüser P, Dohlus M and Rossbach J 2008 Ultraviolet and Soft X-Ray Free-Electron Lasers: Introduction To Physical Principles, Experimental Results, Technological Challenges vol 229 (Berlin: Springer)

[3] Wiedemann H 2007 Particle Accelerator Physics vol 314 (Berlin: Springer)

[4] Wangler T P 2008 RF Linear Accelerators (New York: Wiley)

[5] Egerton R F 1996 Electron Energy-Loss Spectroscopy In The Electron Microscope vol 233 (Berlin: Springer)

[6] Pierce J R 1950 Traveling-wave tubes Bell Syst. Tech. J. 29 390-460

[7] Collins G B 1948 Microwave Magnetrons vol 6 (New York: McGraw-Hill)

[8] Graves W, Kärtner F, Moncton D and Piot P 2012 Intense superradiant $\mathrm{x}$ rays from a compact source using a 
nanocathode array and emittance exchange Phys. Rev. Lett. 108263904

[9] Phuoc K T, Corde S, Thaury C, Malka V, Tafzi A, Goddet J, Shah R, Sebban S and Rousse A 2012 All-optical compton gamma-ray source Nat. Photonics 6 308-11

[10] Ackermann W, Asova G, Ayvazyan V, Azima A, Baboi N, Bähr J, Balandin V, Beutner B, Brandt A and Bolzmann A 2007 Operation of a free-electron laser from the extreme ultraviolet to the water window Nat. Photonics $1336-42$

[11] Andonian G, Stratakis D, Babzien M, Barber S, Fedurin M, Hemsing E, Kusche K, Muggli P, O'Shea B and Wei X 2012 Dielectric wakefield acceleration of a relativistic electron beam in a slab-symmetric dielectric lined waveguide Phys. Rev. Lett. 108244801

[12] Antipov S, Jing C, Kanareykin A, Butler J, Yakimenko V, Fedurin M, Kusche K and Gai W 2012 Experimental demonstration of wakefield effects in a THz planar diamond accelerating structure Appl. Phys. Lett. 100132910

[13] Talebi N, Sigle W, Vogelgesang R and van Aken P 2013 Numerical simulations of interference effects in photonassisted electron energy-loss spectroscopy New J. Phys. 15 053013

[14] Ansoft H 2009 3-D Electromagnetic Simulation Software (Pittsburgh, PA: Ansoft Corp.)

[15] Marsden J E and Weinstein A 1982 The Hamiltonian structure of the Maxwell-Vlasov equations Physica D: Nonlinear Phenom. 4 394-406

[16] Morrison P J 1980 The Maxwell-Vlasov equations as a continuous Hamiltonian system Phys. Lett. A 80 383-6

[17] Seal D C 2012 Discontinous Galerkin methods for Vlasov Models of Plasma (Madison, WI: University of Wisconsin Press)

[18] Dawson J M 1983 Particle simulation of plasmas Rev. Mod. Phys. 55403

[19] Verboncoeur J P 2005 Particle simulation of plasmas: review and advances Plasma Phys. Control. Fusion 47 A231

[20] Salamin Y I 2006 Electron acceleration from rest in vacuum by an axicon Gaussian laser beam Phys. Rev. A 7343402

[21] Wong L J and Kärtner F X 2010 Direct acceleration of an electron in infinite vacuum by a pulsed radially-polarized laser beam Optics Express 18 25035-51

[22] Wong L J, Fallahi A and Kärtner F X 2013 Compact electron acceleration and bunch compression in $\mathrm{THz}$ waveguides Opt. Express 21 9792-806

[23] Marceau V, Varin C and Piché M 2013 Validity of the paraxial approximation for electron acceleration with radially polarized laser beams Opt. Lett. 38 821-3

[24] Hommelhoff P, Sortais Y, Aghajani-Talesh A and Kasevich M A 2006 Field emission tip as a nanometer source of free electron femtosecond pulses Phys. Rev. Lett. 96077401

[25] Hebling J, Yeh K-L, Hoffmann M C and Nelson K A 2008 High-power $\mathrm{THz}$ generation, $\mathrm{THz}$ nonlinear optics, and $\mathrm{THz}$ nonlinear spectroscopy IEEE J. Sel. Top. Quantum Electron. $14345-53$

[26] Taflove A and Hagness S C 2000 Computational Electrodynamics vol 160 (Boston, MA: Artech House Publishers)

Q3 [27] Kern S 1996 Numerische simulation der gyrotronwechselwirkung in koaxialen resonatoren $E U R$ (Luxembourg)

[28] Goplen B, Ludeking L, Smith D and Warren G 1995 Userconfigurable MAGIC for electromagnetic PIC calculations Comput. Phys. Commun. 87 54-86

[29] Weiland T, Bartsch M, Becker U, Bihn M, Blell U, Clemens M, Dehler M, Dohlus M, Drevlak M and Du X 1997 Mafia version 4 Computational Accelerator Physics (New York: AIP) pp 65-70
[30] Burau H, Widera R, Hönig W, Juckeland G, Debus A, Kluge T, Schramm U, Cowan T E, Sauerbrey R and Bussmann M 2010 PIConGPU: A fully relativistic particlein-cell code for a GPU cluster IEEE Trans. Plasma Sci. 38 2831-9

[31] Hesthaven J S and Warburton T 2002 Nodal high-order methods on unstructured grids: I. Time-domain solution of Maxwell's equations J. Comput. Phys. 181 186-221

[32] Hesthaven J and Warburton T 2004 High-order nodal discontinuous Galerkin methods for the Maxwell eigenvalue problem Phil. Trans. R. Soc. A 362 493-524

[33] Kabakian A V, Shankar V and Hall W F 2004 Unstructured grid-based discontinuous Galerkin method for broadband electromagnetic simulations J. Sci. Comput. 20 405-31

[34] Stannigel K, Niegemann J and Busch K 2009 Discontinuous Galerkin time-domain computations of metallic nanostructures Opt. Express 17 14934-47

[35] Busch K, König M and Niegemann J 2011 Discontinuous Galerkin methods in nanophotonics Laser Photonics Rev. 5 773-809

[36] Jacobs G and Hesthaven J 2006 High-order nodal discontinuous Galerkin particle-in-cell method on unstructured grids J. Comput. Phys. 214 96-121

[37] Gjonaj E, Lau T, Schnepp S, Wolfheimer F and Weiland T 2006 Accurate modelling of charged particle beams in linear accelerators New J. Phys. 8285

[38] Gjonaj E, Lau T and Weiland T 2007 Conservation properties of the discontinuous Galerkin method for Maxwell equations Electromagnetics in Advanced Applications, 2007. ICEAA 2007. Int. Conf. on (IEEE) pp 356-9

[39] Stindl T, Neudorfer J, Stock A, Auweter-Kurtz M, Munz C and Roller S Schneider R 2011 Comparison of coupling techniques in a high-order discontinuous Galerkin-based particle-in-cell solver J. Phys. D: Appl. Phys. 44194004

[40] Gjonaj E and Weiland T 2010 Particle based PWFA simulations using a Discontinuous Galerkin approach Electromagnetics in Advanced Applications (ICEAA), 2010 Int. Conf. on (IEEE) pp 604-7

[41] Webb J P 1999 Hierarchal vector basis functions of arbitrary order for triangular and tetrahedral finite elements IEEE Trans. Antennas Propag. 47 1244-53

[42] Shankar V, Hall W F and Mohammadian A H 1989 A timedomain differential solver for electromagnetic scattering problems Proc. IEEE 77 709-21

[43] Vial A, Grimault A-S, Macías D, Barchiesi D and de la Chapelle M L 2005 Improved analytical fit of gold dispersion: application to the modeling of extinction spectra with a finite-difference time-domain method Phys. Rev. B 71 085416

[44] Darden T, York D and Pedersen L 1993 Particle mesh Ewald: an $\mathrm{N} \cdot \log (\mathrm{N})$ method for Ewald sums in large systems J. Chem. Phys. 98 10089-92

[45] Deserno M and Holm C 1998 How to mesh up Ewald sums. I. A theoretical and numerical comparison of various particle mesh routines J. Chem. Phys. 109 7678-93

[46] Greengard L and Rokhlin V 1987 A fast algorithm for particle simulations J. Comput. Phys. 73 325-48

[47] Alvarez J, Angulo L, Bretones A R and Garcia S G 2012 A spurious-free discontinuous Galerkin time-domain method for the accurate modeling of microwave filters IEEE Trans. Microw. Theory Tech. 60 2359-69

[48] Klees R 1996 Numerical calculation of weakly singular surface integrals J. Geod. 70 781-97

[49] Fowler R H and Nordheim L 1928 Electron emission in intense electric fields Proc. R. Soc. A 173-81

[50] Becker W, Grasbon F, Kopold R, Milosevic D, Paulus G and Walther H 2008 Above-threshold ionization: from classical features toquantum effects 
[51] Swanwick M, Keathley P, Kartner F and Velásquez-García L 2013 Ultrafast photo-triggered field emission cathodes using massive, uniform arrays of nano-sharp high-aspect-ratio silicon structures Solid-State Sensors, Actuators and Microsystems (TRANSDUCERS \& EUROSENSORS XXVII),
2013 Transducers \& Eurosensors XXVII: The 17th Int. Conf. on (IEEE) pp 2680-3 


\section{QUERY FORM}

Journal: Journal of Physics B: Atomic, Molecular and Optical Physics

Author: A Fallahi and F Kärtner

TiTLE: Field-based DGTD/PIC technique for general and stable simulation of interaction between light and electron bunches

ARTICLE ID: jpb500876

The layout of this article has not yet been finalized. Therefore this proof may contain columns that are not fully balanced/ matched or overlapping text in inline equations; these issues will be resolved once the final corrections have been incorporated.

SQ1

Author: Please be aware that the colour figures in this article will only appear in colour in the online version. If you require colour in the printed journal and have not previously arranged it, please contact the Production Editor now.

\section{Page}

Q1

We have been provided funding information for this article as below. Please confirm whether this information is correct. European Research Council: 609920.

\section{Page 12}

Q2

We have been provided funding information for this article as below. Please confirm whether this information is correct. Fundername: European Research Council Funderid: 10.13039/501100000781 Grantnumbers: 609920

\section{Page 12}

Q6

Please check the details for any journal references that do not have a link as they may contain some incorrect information.

Page 13

Q3

Please provide the volume number and the page range or article number in reference [27].

\section{Page 13}

Q4

Please provide the volume number in reference [49].

\section{Page 13}

Q5

Please check if this reference is to a book, journal conference proceedings or other and supply the complete details as appropriate. 